\title{
AN EFFICIENT LOAD SHEDDING ALGORITHM FOR RADIAL SYSTEMS
}

\author{
P.S. NAGENDRA RAO AND K.S. PAPA RAO \\ Department of Electrical Engineering, \\ Indian Institute of Science, \\ Bangalore 560012 , India. \\ E.mail: nagendra@ee.iisc.ernet.in
}

\begin{abstract}
Distribution Automation is being adopted by several utilities to improve their operation. The importance of efficient load shedding algorithms in automated distribution systems has been known for some time. Yet there has been very little work in this area. This paper formulates this problem as a simple graph search problem. A simple and effective algorithm has been developed to determine the loads to be shed in order to overcome the abnormal operating conditions in the system.
\end{abstract}

\section{INTRODUCTION}

Load shedding may be the only option under abnormal loading conditions, wherein the system conditions may be so severe that they cannot be completely set right by reconfiguration of the network. Some service restoration algorithms may reach unacceptable configurations and some zones have to be shed, to reach an acceptable configuration. In such situations, algorithms are needed to decide which particular loads are to be shed, so that service disruptions are reduced without sacrificing the service quality. Not many algorithms have been reported so far this problem, even though load shedding algorithms are required in several situations. Aoki et al [1] describe a load curtailment procedure as part of a service restoration algorithm considering a violation vector with current capacity and voltage drop violations as components. A quantity called effective length of remaining violation is defined in [1]. Loads in the end sections of the violating feeders that have the smallest value of this quantity are curtailed. This metric does not appear to be appropriate, since it does not seem to use the fact that current capacity violation in a line, can be eliminated only by shedding a load that is getting its supply through that line. The choice of loads for curtailment is dependent on an arbitrary constant. The metric can also be misleading due to the use of the product of load and remaining violation, as this could result in a small load that does not help in removing the violation being curtailed. Load shedding has also been considered by Sarma et al [2] in a slightly different context. They consider systems with switchable capacitors and on-load tap changers. For voltage drop violations, if the load point with violation does not have a switchable capacitor, it will be shed. If such a load point happens to be of a high priority, a nearby load point of low priority is chosen for shedding. For current capacity viola-

$0.7803-7651-\mathrm{X} / 03 / \$ 17.00$ @20031FEE tion at a component, a low priority load at a point beyond that component is shed. However, the steps to be taken when several current capacity and voltage drop violations are simultaneously present are not specified in this paper. The priority restoration algorithm of Teo et al [3] uses the load shedding method to remove the lower priority loads at any bus. Reconfiguration at the sub-station is considered here. Each bus in the network is given a priority number which reflects the importance of its load. If the total load of a dead island is greater than the capacity, loads of the lowest priority is done by a best-fit method. The load of a dead bus selected is disconnected but the bus still remains connected. At the end, an attempt is made to restore the loads that were left out due to their low priority values. The restored sequence starts from the largest load in the highest priority group, followed by the next largest load in the same priority group. It is easy to see that the emphasis in this work is on restoration and not on load shedding. Considering all these attempts, one can easily infer that, the load shedding problem has not received the attention it deserves. These existing algorithms are too nave and are neither comprehensive nor efficient. Here, we consider load shedding as an independent problem and assess the issues to be considered in developing efficient algorithims for solving the problem. We propose an algorithm which requires-a-limited systematic search effort to determine the optimal load shedding.

\section{BASIS OF THE ALGORITHM}

As the system is radial, every zone is supplied through only one of its incident lines. Such an input line is called source line of the zone. Currents may be leaving the zone through several lines. Such lines are called the sink lines of the zone. All the lines and loads that are getting their supply through a line, are called followers of that line. Followers of all sink. lines of a zone, put together are called followers of that zone. A zone with no followers is a leaf zone. It may be noted that all the zones that follow a zone are not necessarily followers of all the lines in the zone. Shedding loads supplied by a feeder does not affect the operating conditions in areas supplied by other feeders. Hence, without loss of generality, in the discussions to follow, we limit ourselves to a single feeder.

Violations to be dealt with are, line ampacity violations and voltage drop violations at the load points. When both current capacity violations and voltage drop violations are simultane- 


\section{TENCON $2003 / 772$}

ously present, current capacity violations are eliminated first. This is because, load shedding to remove a line ampacity violation has to be performed only from the zones following that line, whereas shedding load anywhere in the feeder reduces the voltage drops throughout the network. Thus several of the voltage drop violations may get eliminated/reduced while loads are shed to remove the line ampacity violations. When several line capacity violations are present within a zone, violations in those lines that are closer to the leaf load points have to be handled first. It is easy to see that line capacity violations in zones closer to the leaf zones must be handled before considering zones that are farther away from the leaf zones. In the rare possibility of current capacity violations occurring in a leaf zone, that leaf zone has to be shed before handling other current capacity violations. For handling voltage drop violations in a zone, it is easy to see that shedding its farthest follower zone gives maximum relief. Thus, while shedding zones following a line with capacity violation, those that also happen to follow a zone with voltage drop violation, are given preference. Only the leaf zones must be shed. Shedding any other zone, results in switching off of several zones from supply simultaneously, and is to be avoided. The set of leaf zones alters as leaf zones are shed. A non-leaf zone becomes a leaf zone, when all the zones following it are shed.

The load shedding algorithm proposed here is designed to have three stages. In the first stage, loads are shed for eliminating current capacity violations. In the second stage, the remaining voltage drop violations are eliminated. Finally, in the third stage, we check whether supply to some of the zones shed, can be restored without causing violations again.

Starting at the set of leaf zones, the algorithm checks for amapcity violations in every zone. A zone is picked up for violation checking only after ensuring that all its follower zones are violation free (or are made free from violations by shed(ing). Current capacity violation in any line is eliminated by shedding some appropriate leaf zone followers of that line. Within a zone, with several ampacity violations, violations in lines closer to the sink lines are eliminated first. By the end of the first stage, the severity of the voltage drop violations gets reduced. While there are leaf zones with voltage drop violations, the leaf zone containing the load point with the maximum voltage drop violation is shed. Otherwise, the leaf zone follower of the zone with voltage drop violation, that gives maximum relief to the voltage drop violation being handled, per unit of current shed, is selected for shedding. Thus, if $w$ is the load point with the highest voltage drop in the zone under consideration, leaf zone followers of the branching point immediately preceding $w$ are preferred for shedding, over other leaf zone followers of the same zone. It is possible that more load than necessary, may be shed in some cases. Hence, in the third stage, the possibility of restoring some of the zones shed is considered. Priority of the loads is not taken into account in the proposed algorithm. However, it can easily be incorporated by enforcing that the leaf zones having high priority loads should not be shed, as long as any other option is available for eliminating the same violation.

\section{LOAD SHEdDING ALGORITHM (LSA)}

The steps of the new load shedding algorithm are as follows: Stage 1 (eliminating ampacity violations)

1.Initialise the set of zones under consideration, I, to the set of leaf zones

2.Pick a zone $z \epsilon \mathrm{I}$, with ampacity violations. If none can be picked, go to step 5 .

3.Among the lines in $\mathrm{z}$ having current capacity violations, pick a line 1 , that is closest to the sink lines.

If no such line can be picked, go to step 2 .

4. While the capacity violation in 1 is persisting,

from the leaf zones following l, shed a zone, giving first priority to zones containing the load point with the maximum voltage drop violation, and second priority to zones that follow a follower zone of 1 having voltage drop violation. Update network voltages and currents

Go to step 3.

5.Find the set of leaf zones I', assuming all the zones in I and their followers are shed.

If I' is empty, go to step 6.

Else, set I = I' and go to step 2.

Stage 2 (eliminating voltage drop violations)

6. While there are leaf zones with voltage drop violations, shed the leaf zone with the maximum voltage drop violation. Update network voltages and currents.

Repeat.

7. If there are no voltage drop violations, go to step 8 .

Else, pick a zone $\mathrm{z}$ with voltage drop violation and shed one of its leaf zone followers, such that the ratio of relief in the voltage drop obtained in the worst load point of zone $\mathrm{z}$ to the amount of load shed, is maximized.

Update network voltages and currents. Go to step 6.

Stage 3 (back tracking)

8.Declare the set of zones shed as the affected area As, and rest of the area as healthy, Hs, and solve the service restoration problem for restoring as much of As as possible, using a modified BLTA of Aoki et al [4], with voltage drop constraints included.

\section{Performance eVAluation}

The performance of the proposed algorithm has been studied considering several systems. Here, we provide a sample by considering a 470 node distribution feeder. This is a $13.2 \mathrm{kv}$ system and a reduced system has been derived by merging adjacent nodes where ever possible. A schematic diagram of the system is given in Figl. The load points in the system are given unique numbers. The edge preceeding a node is given the same number as the node. The resistance of the lines and the currents at various points are available in [5].

The load shedding algorithm is used when the total load current in the feeder is686.86 A. Corresponding to this load, current current capacity constraint is violated in lines $2,5,10,28,57,72$ and 97 . The permissible voltage drop in the 


\section{Power Transmission and Distribution / 773}

feeder is $250 \mathrm{~V}$. At this load the voltage constraint is violated at a number of nodes. Since each line in the diagram represents actually a zone, the line/node numbers also represent the zone numbers.

Starting at leaf zones, all zones are tested for current capacity violations. No ampacity violations are found til a stge with $I=97$ is reached. where the drop is $263.8 \mathrm{~V}$. Thus switch 337 is opened.

This leaves 231 as the only leaf zone with voltage drop violations and the maximum drop in that zone is 251.3 volts. Switxh 230 is opened and all voltage drop violations are removed, completing stage- 2 .

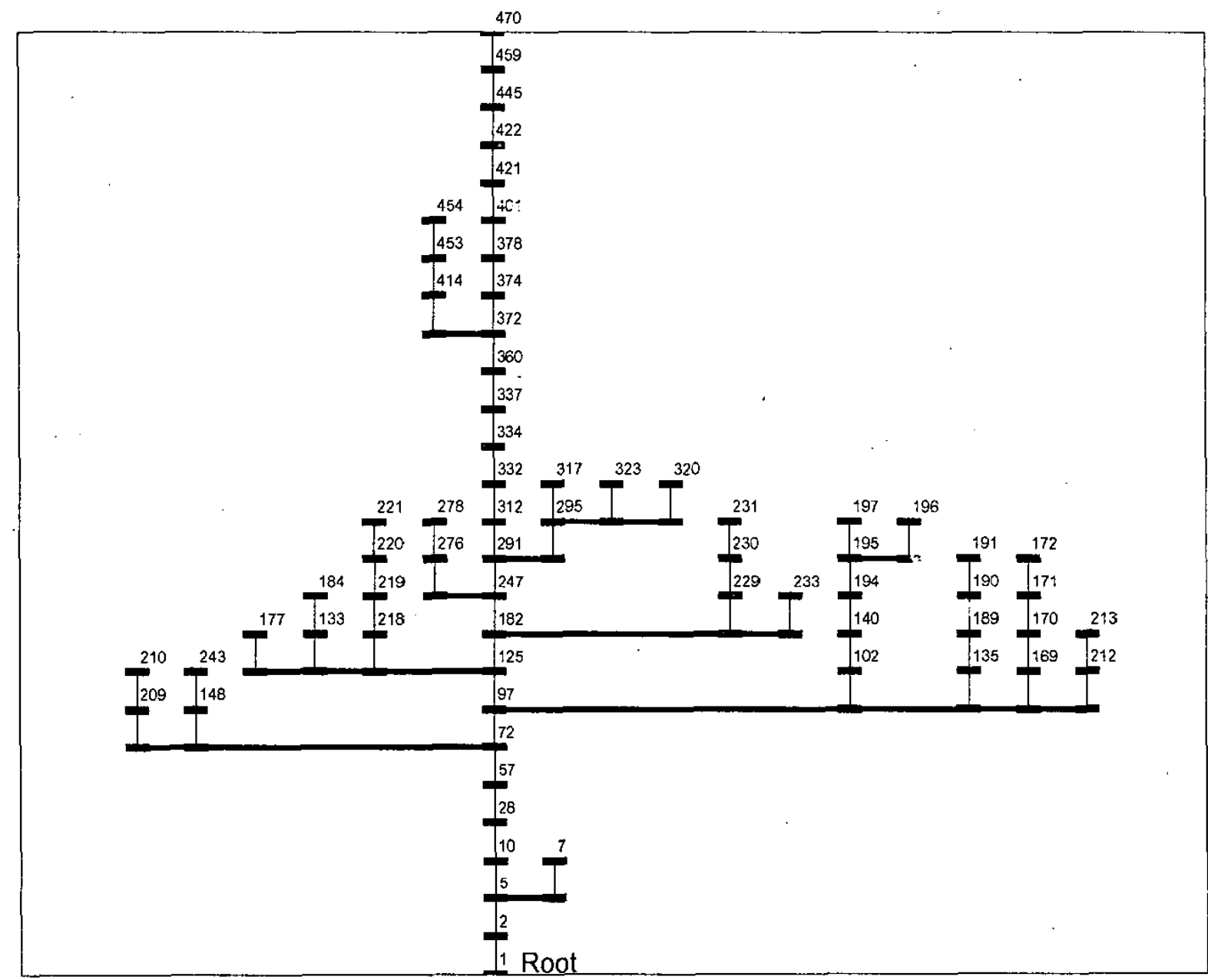

Figure 1. Single line diagram of the reduced 470 node feeder

The line with the current capacity violation considered is 97 , where current flow exceeded cpacity by $100.7 \mathrm{~A}$.

Among the leaf zones following the line 97, the maximum voltage drop violation is in zone 470 , where the maximum drop is 459.3 volts. Thus zone 470 is shed by opening switch 445 first. Continuing this process a number of ones are shed to complete the stage 1 .

In stage- 3 it is checked to see, if any of the shed loads can be picked. There are 5 cut-switches at this stage. They are 230 , 276, 295 and 337. Moving any cut-switch results in fresh violations, hence supply cannot be restored to any of the shed loads.

At the time the algorithm terminates, the total load fed by the feeder is $451.5 \mathrm{~A}$.

\section{CONCLUSION}

By the end of stage-1, leaf zones with voltage drop violations are 191,231 and 360 . The maximum violation is at zone 360

The importance of developing efficient algorithms for load shedding for use in automated distribution systems has been 
highlighted. Issues to be considered while developing such algorithms are discussed. A new algorithm for load shedding has been developed which can eliminate both ampacity and voltage drop violations. The new algorithm is simple to implement and involves only a limited systematic search. The practicability of the algorithm is demonstrated by illustrating its use on some realistic systems.

\section{REFERENCES}

[1] K. Aoki, N. Nara, M. Itoh and T. Satoh and H. Kuwabara. A new algorithm for service restoration in distribution systems. IEEE PWRD, 4(3):1832-1839, 1989.

[2] N.D.R. Sarma, S. Ghosh, K.S. Prakasa Rao, and M. Srinivas. Real time service restoration in distribution networks - a practical approach. IEEE PWRD, 9(4):2064-2070, 1994.

[3] C.Y. Teo and H.B. Gooi. Restoration of electrical power supply through an algorithm and knowledge based system. Electric Power Systems Research, 29:171-180, 1994.

[4] K. Aoki, H. Kuwabara, T. Satoh, and M. Kanezashi. Outage state optimal load allocation by automatic sectionalizing switches operation in distribution systems. IEEE PWRD, 2(4):1177-1185, 1987.

[5] K. S. Papa Rao, Reconfiguration Algorithms for Distribution Automation, M.Sc. thesis, Indian Institue of Science, 1995 